\title{
Comfortable
}

retraction

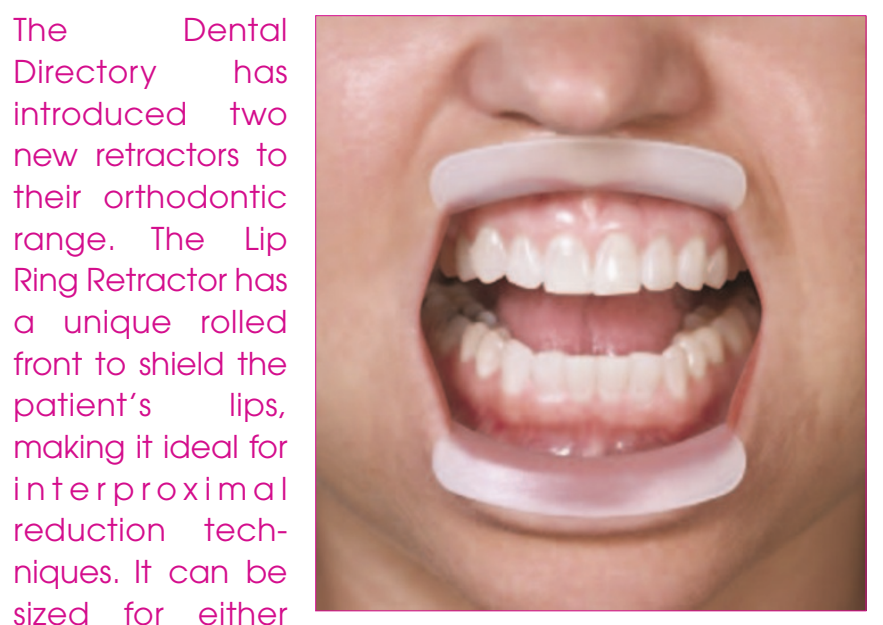

adults or children and is made of high quality medical grade plastic. The working field can be accessed with ease and minimal discomfort for the patient, making it ideal for bonding and intra-oral photography. The second new product is a photo lip retractor, Retract EEZ, which can be used as an aid for occlusal photography as well as during bleaching procedures since its design moves the patient's lips to allow maximum exposure. It can be cold or heat sterilised up to $143^{\circ} \mathrm{C}$.

\section{Promoting better posture}

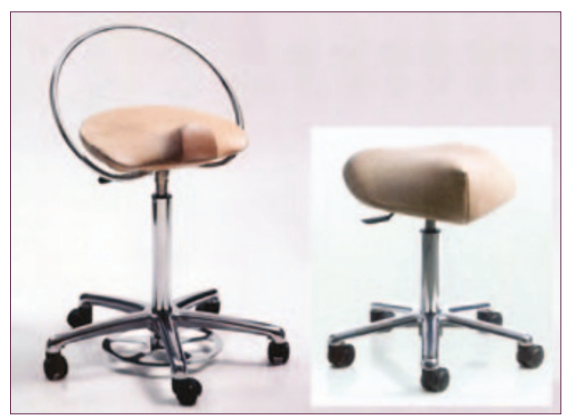

Greater comfort and improved posture feature in Evident's new DynamoSeat and InCharge stools which actively promote better posture for the whole team by encouraging a correct sitting posi-

tion. While seated, backs can be upright and better balanced, and legs less restricted, offering improved circulatory benefits and greater mobility around the surgery. The company also offers the Orascoptic loupes with a full range of lightweight and magnification options with a high-resolution quality image. The 'silent-running' Zeon Illuminator provides perfect illumination to enhance the magnification experience.

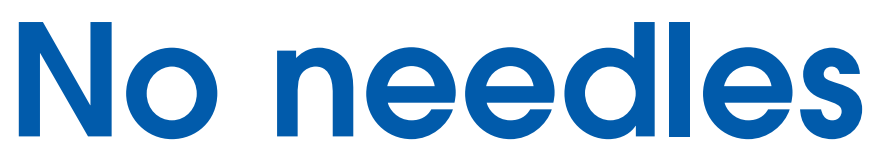

\section{solution}

Oraqix periodontal gel (lidocaine, prilocaine) from Dentsply Ltd is a non-injectable dental local anaesthetic. It is indicated in adults for localised anaesthesia in periodontal pockets, and for diagnostic and treatment procedures such as probing, scaling and/or root planing (SRP).

There is no need for a needle for those who are phobic - and it is a quick option to provide pain relief during SRP for those who normally have to go without. Oraqix is applied to the periodontal pocket with a special patient-friendly, ergonomic Oraqix Dispenser, developed by Dentsply and takes effect in around 30 seconds.

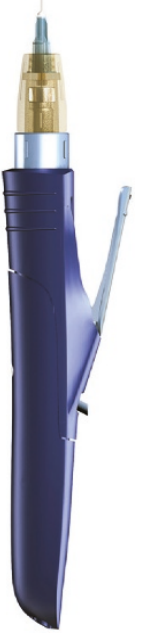

\section{Hydrating coating technology}

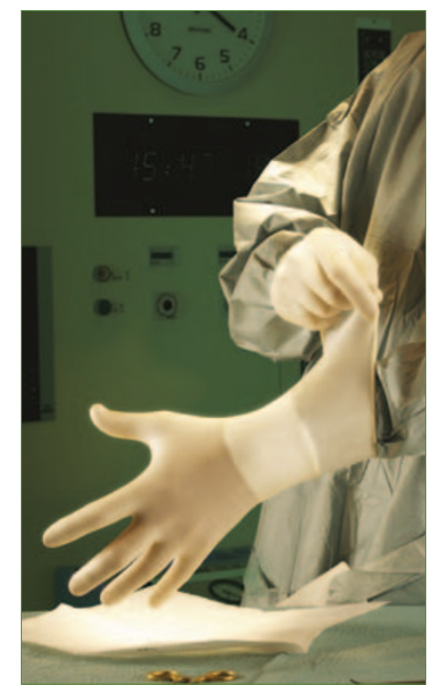

Ansell Healthcare Europe, has announced the launch of HydraSoft, a new hydrating coating technology for surgical gloves. Designed to retain moisture and rehydrate wearers' skin during use, HydraSoft has been specifically developed for clinicians' hands, to help maintain the skin's natural barrier function. Composed of neutral, odourless ingredients in the glove's inner lining, the product is designed to protect skin from drying, cracking and penetration by foreign substances. It contains a skin-friendly, humectant moisturiser combined with dimethicone, which forms a protective barrier and prevents the skin from drying out. Ansell plans to first introduce HydraSoft coating technology into its bestselling powder-free latex surgical glove, Gammex PF. The introduction of HydraSoft has also permitted a modified formulation, resulting in a softer, suppler glove scoring highly on sensitivity. The company plans to integrate HydraSoft into other products within its medical glove range. 